\title{
ON THE STIMULUS DUTY CYCLE IN STEADY STATE VISUAL EVOKED POTENTIAL
}

\author{
JOHN J. WILSON \\ School of Computer Science and Electronic Engineering, University of Essex, Colchester, CO4 3SQ, \\ United Kingdom
}

RAMASWAMY PALANIAPPAN

Department of Engineering, Faculty of Science and Engineering, University of Wolverhampton, Telford Innovation Campus, TF2 9NT, United Kingdom

Corresponding author:

Dr Ramaswamy Palaniappan

Department of Engineering

Faculty of Science and Engineering, Room SC035

University of Wolverhampton

Shifnal Road, Priorslee

TF2 9NT, Telford, UK

Tel/Fax: 44-(0)-1902-323875/323843

Email: palani@wlv.ac.uk

\begin{abstract}
Brain-computer interfaces (BCI) are useful devices that allow direct control of external devices using thoughts, i.e. brain's electrical activity. There are several BCI paradigms, of which steady state visual evoked potential (SSVEP) is the most commonly used due to its quick response and accuracy. SSVEP stimuli are typically generated by varying the luminance of a target for a set number of frames or display events. Conventionally, SSVEP based BCI paradigms use magnitude (amplitude) information from frequency domain but recently, SSVEP based BCI paradigms have begun to utilize phase information to discriminate between similar frequency targets. This paper will demonstrate that using a single frame to modulate a stimulus may lead to a bi-modal distribution of SSVEP as a consequence of a user attending both transition edges. This incoherence, while of less importance in traditional magnitude domain SSVEP BCIs becomes critical when phase is taken into account. An alternative modulation technique incorporating a 50\% duty cycle is also a popular method for generating SSVEP stimuli but has a unimodal distribution due to user's forced attention to a single transition edge. This paper demonstrates that utilizing the second method results in significantly enhanced performance in information transfer rate in a phase discrimination SSVEP based BCI.
\end{abstract}




\section{Introduction}

Electroencephalogram (EEG) is the recording of brain's electrical activity and analysis of EEG signals is useful for identifying problems with the neurological and mental system such as sleep stages [1], alcoholism [2] and even biometrics [3]. Recently, EEG has been used in the design of braincomputer interfaces (BCIs) [4]. BCI systems allow direct control of external devices (for example, controlling a wheelchair, moving computer cursor or selecting menus on screen) using thought patterns from the brain recorded with EEG. Obviously, such BCI systems will be useful for the severely disabled (such as those with locked-in syndrome). In recent years, other applications of BCI such as gaming and virtual reality have been developed. Among numerous BCI paradigms, steady state visual evoked potential (SSVEP) is a highly viable and popular paradigm for an online BCI as compared to mental tasks [5] or motor imagery [6] due to its simple paradigm requiring low setup time, little or no training and robust presence in most user's EEG. The SSVEP is evoked when a subject is exposed to repeated visual stimulation such that the reaction to a subsequent stimulus occurs before the effect of the previous stimulus has subsided. SSVEP based BCI's are amongst the highest performing BCIs in terms of Information Transfer Rate (ITR) with bit rates of over $90 \mathrm{bit} / \mathrm{min}$ reported [7].

Typically SSVEP is elicited through presentation of visual stimuli flashing at unique frequencies via use of traditional visual display units (VDUs) such as CRT or LCD monitors or custom built LED arrays. Commonly SSVEP is detected and classified in EEG through Fourier based frequency analysis utilizing the magnitude of the SSVEP response alone. This is possible as the frequency of the flashing target object is resonated in the recorded SSVEP and detection of the frequency in SSVEP allows the recognition of the object focused by the user. However, unlike other visually synchronous BCI paradigms such as P300 [7], SSVEP is fundamentally constrained in the number of unique targets that can be presented in the system due to limitations of 1) the base adapter rate of the VDU [9] and 2) the time-frequency uncertainty relationship of Fourier analysis [10].

To counteract these limitations and allow a higher number of targets, the targets can be encoded and classified by phase and frequency $[11,12]$ rather than frequency alone, requiring no increase in VDU frequency or Fourier time resolution. Mixed frequency and phase coded SSVEP BCI's with up to 15 targets have been demonstrated, however so far ITR's are comparable to frequency only systems $[13,14]$.

In this paper, it will be demonstrated that the transition edges occurring in stimulus modulation using a single frame may lead to a bi-modal distribution of SSVEP as a consequence of a user attending both transitions (i.e. ON-OFF and OFF-ON) edges. This incoherence, while of less importance in traditional magnitude domain SSVEP BCIs becomes critical when phase is taken into account. Fifty percent duty cycle is also a popular alternative method for generating SSVEP stimuli and has a unimodal distribution due to user's forced attention to a single transition edge. This paper demonstrates that utilizing the second method results in significantly enhanced performance in information transfer rate in a phase discrimination SSVEP based BCI. The rest of the paper is organized as follows: Section 2 discusses both the simulation methods, while the experimental study is described in Section 3. The next section describes the analysis of the SSVEP and the results are given and discussed in Section 5. The final section concludes the paper.

\section{SSVEP Stimulus Generation}

SSVEP targets are generated by a luminosity change that occurs at set intervals and is directly related to the base adapter rate of the VDU. A typical LCD monitor has a refresh rate of $60 \mathrm{~Hz}$ allowing for targets that flash at integer divisors of this rate e.g. $20 \mathrm{~Hz}, 12 \mathrm{~Hz}, 6.67 \mathrm{~Hz}$ etc but avoiding harmonics. For example, if $20 \mathrm{~Hz}$ is used for one target, then $10 \mathrm{~Hz}$ should be avoided. Flashing frequency of less than $6 \mathrm{~Hz}$ is not used for SSVEP as the flashes become too slow to evoke a steady state response. In 
the next section, stimuli construction will be discussed in terms of pattern reversal of a computer generated image, however the discussion is synonymous with LEDs flicking on and off.

\subsection{Single frame duty cycle - Method A}

In this method of stimulus generation (subsequently referred to as Method A) a luminosity alteration occurs in a single frame, before returning to the base state in the subsequent frame. Thus there are two transitions, from the base to alternate state and vice versa.

\section{2. $50 \%$ duty cycle - Method B}

In this method (subsequently referred to as Method B) there is no 'base' state for a target, instead the target alternates between states at the desired interval. Thus there is a single transition at each change.

Methods A and B are adopted approximately equally in a recent survey of SSVEP BCI stimulation methods [15]. Figure 1 depicts how each method is constructed on a frame by frame basis for an example of $12 \mathrm{~Hz}$ stimulus. It can be seen that there will be a discrepancy in phase if a user attends either the leading edge or falling edge of the transition in Method A. In contrast, Method B incorporates only a single transitional edge as the luminosity of the target remains changed for the duration of the modulation cycle allowing the user to only attend a single, phase coherent transition over multiple cycles.

The experimental study here compares methods A and B in terms of overall SSVEP response, phase coherency and ITR via a 35 target SSVEP BCI utilizing five different frequencies (detailed in the next section) using all possible phase segments (it should be noted that a higher number of targets have been studied here using mixed phase and frequency coding as compared to previous works [11, 12]). Figure 2 shows the simulation paradigm for both methods for these frequencies.

\section{Methods}

\subsection{Experimental Setup}

\section{Stimulation Engine}

The stimulation engine was presented using a standard 24 inch widescreen $60 \mathrm{~Hz} \mathrm{LCD}$ monitor and was created in Mathworks Matlab using the Psychophysics toolbox. The engine presented 35 targets of pattern reversing checkerboards. Each target was 250 x 200 pixels in size and contained $25 \times 25$ pixel individual checks as shown in Figure 3. Stimuli flashing frequencies used were $6.66 \mathrm{~Hz}, 7.5 \mathrm{~Hz}$, $8.57 \mathrm{~Hz}, 10 \mathrm{~Hz}, 12 \mathrm{~Hz}$ and targets were arranged so that no similar frequency stimuli were situated adjacently.

\section{Phase Coding}

Phase encoding of targets is achieved by shifting the frame at which the stimulus alternates. The number of unique phase targets, $M(f)$ is a function of target frequency, $f$ and the refresh rate of the VDU, $f_{v d u}$ and is determined by Eq. (1):

$$
M(f)=f_{v d u} / f .
$$


where $M=1,2,3 \ldots . . N$. The phase separation between each target, $\Delta \phi s(f)$ at any particular stimulus frequency $f$ is calculated by (2):

$$
\Delta \phi s(f)=\frac{360^{\circ}}{M(f)}
$$

As can be deducted, lower frequencies have more available targets but the phase separation between them becomes smaller.

\subsection{Experimental Paradigm}

Five subjects ${ }^{*}$ completed ten sessions in total (five with stimulation Method A and five using Method B). Each session consisted of 35 trials corresponding to attending every target in the system. A trial consisted of a two second cueing/resting period in which a red cross indicated the target to subsequently attend. During this period the targets did not flash. The subject was then required to attend the cued target for the subsequent six seconds in which all targets flashed at their given rates and phases. To ensure the subject was attending the cued target two random characters appeared within the target area which the subject reported during the subsequent cueing/resting period. Each session incorporated two 10s rest after trials 12 and 24 and there was two minutes break between sessions giving a total experimental time of one hour for each subject.

\subsection{EEG Acquisition}

EEG was recorded using three gold electrodes, two in a bipolar configuration at locations $\mathrm{Oz}$ and $\mathrm{PO} 3$ according to the international 10/20 system and a final electrode at the forehead (Fpz) serving as ground. Impedances were kept below $5 \mathrm{k} \Omega$ through use of conductive paste. The EEG biosignal amplifier employed was the g.BSamp coupled to the g.16sys subsystem (g.tec, Guger Technologies) incorporating a hardware bandpass from $2-30 \mathrm{~Hz}$, with a notch filter at $50 \mathrm{~Hz}$ (UK AC power frequency). To ensure that no drift could occur in the signal, EEG was acquired in single point hardware timed mode using National Instruments LabView. Due to the entire system being implemented on a single PC for future online use the sampling rate was restricted to $60 \mathrm{~Hz}$.

\section{Analysis}

\subsection{Single Cycle Analysis}

Single cycle analysis is a useful tool allowing insight into the individual visual evoked potential flash events that make up the SSVEP response over an entire stimulation epoch [16]. Rectangular sliding windows corresponding to single cycle sample lengths of the five frequencies used stimuli (5pts $12 \mathrm{~Hz}, 6 \mathrm{pts}-10 \mathrm{~Hz}, 7 \mathrm{pts}-8.57 \mathrm{~Hz}, 8 \mathrm{pts}-7.5 \mathrm{~Hz}, 9 \mathrm{pts}-6.66 \mathrm{~Hz}$ ) were sequentially slid over the entire six second epoch and submitted to a Fast Fourier Transform (FFT) operation individually. The resulting arrays of complex frequency components $X$, corresponding to each of the five fundamental repetition rates are then utilized in the following algorithms.

\section{Magnitude Squared Coherence}

Magnitude Squared Coherence (MSC) [17] is the ratio of the grand average summed SSVEP power to average amplitude of the single cycles and calculated by Eq. (3) for any of the given targets, $T_{1}, T_{2}, \ldots, T_{N}:$

\footnotetext{
${ }^{*}$ Since all subjects completed both Method A and Method B and comparison of performance was for each subject, the mean age, sex, formal education, etc do not affect the comparative results.
} 


$$
\operatorname{MSC}\left(T_{n}\right)=\frac{\left|\frac{1}{C} \sum_{c=1}^{C} X_{c}\left(T_{n}\right)\right|}{\frac{1}{C} \sum_{c=1}^{C}\left|X_{c}\left(T_{n}\right)\right|} \quad \text { for } n=1, \ldots . N
$$

where $C$ is the number of cycles in the window for each frequency. MSC tends toward zero when SSVEP response is low and toward one when SSVEP response is high.

\subsection{Deviation from Expected Phase}

\section{Mean Expected Phase Deviation}

The mean absolute difference in phase angle between successive elicited expected phase angles $(\Delta \phi a)$ and the corresponding stimulation phase difference $(\Delta \phi s)$ results in the mean phase deviation $\bar{\phi}$ at any given frequency $f$ and is calculated as in Eq. (4):

$$
\bar{\phi}(f)=\frac{1}{N} \sum_{i=1}^{N}\left|\Delta \phi s_{i}(f)-\Delta \phi a_{i}(f)\right|,
$$

where $N$ is the number of targets (i.e. 35 in this study). Ideally, values $\bar{\phi}$ should be as close to zero as possible. A value of zero indicates that recorded phase angles and target stimuli phase angles are identically dispersed.

\subsection{Classification Performance}

\section{Training, Testing and Cross Validation}

The first four trials for each target of each stimulation method were used as a training set to construct a calibrated phase response by averaging the orthogonal components of the result of an FFT over the entire epoch at the bin corresponding to the target frequency. The final remaining trial is used for testing and the five coefficients from an FFT at each fundamental frequency projected onto each of the 35 calibrated phase angles. The target corresponding to the resulting maximal projected value was then selected as the attended target.

The trials were then re-labeled as training or testing to carry out a fivefold cross validation averaging the classification accuracy rate at each stage to arrive at a global classification accuracy rate for each subject.

\section{Information Transfer Rate}

Bit rate is a standard measure of communication systems and can be used to evaluate BCI performance. It depends on the number of available targets $N$ and the classification accuracy rate $P$ and is calculated by Eq. (5) [18]:

$$
\text { Bitrate }=\log _{2} N+P \log _{2} P+(1-P) \log _{2} \frac{1-P}{N-1}
$$

The ITR in bits per minute (bit/min) can then be calculated by multiplying the bits rate by the number of targets that can be selected in 60 seconds. 


\section{Results}

Table 1 compares the two stimulation methods for each subject in terms of three parameters: MSC, $\bar{\phi}$ and ITR (as discussed in the Section 4). For each subject ITR rates were increased when using Method B compared to Method A. Training and testing classification was carried out using only the first two seconds of stimulation data. Allowing for $0.5 \mathrm{~s}$ time for a subject to change gaze between targets gives targets per minute rate of 24 from which ITR was calculated according to Eq. (5). Overall subjects' ITR's were significantly increased between method A to method B from $38.79 \mathrm{bit} / \mathrm{min}$ to $67.62 \mathrm{bit} / \mathrm{min}$ (t-test significance, $p=0.009$ ). This inversely correlated with mean phase deviation from expected $\bar{\phi}$ which was reduced for each subject and overall was significantly smaller at $40.01^{\circ}$ for Method B and $49.54^{\circ}$ for Method A (t-test significance, $p=0.018$ ). Interestingly there was no significant difference in extent of SSVEP response between the methods with MSC values being nearly equal.

Figure 4 depicts phase histograms for subject 2 over the six seconds stimulation period attending the $12 \mathrm{~Hz}$, phase segment five stimulus. Each bin is $35^{\circ}$ wide corresponding to one half of $\Delta \phi s$ for the $12 \mathrm{~Hz}$ frequency stimulus. The Method A histogram depicted in Figure 4(a) shows a distribution spread over three bins compared to the much tighter distribution of Method B which has its modal vector distribution within an individual bin. This example is consistent with the hypothesis that in the case of method A the subject may have attended each transition edge over the duration of the stimulation period causing SSVEP response to be dispersed by an amount equal to the phase separation of the given target, in this example $\Delta \phi s f_{12}=72^{\circ}$. When we add this to the mean recorded phase separation $\bar{\phi}$ of subject 3 from Table 1 we arrive at a SSVEP vector dispersal of $72^{\circ}+36.48^{\circ}=$ $108.48^{\circ}$ degrees which corresponds almost exactly to the distribution over three bins widths $(35 \times 3=$ $105^{\circ}$ ) depicted in Figure 4(a).

Figure 4(b) depicts the corresponding $12 \mathrm{~Hz}$ calibration phase maps for subject 3 over all trials. Here we can note the impact of the inconsistent distribution resulting from method A in Figure 4(a) where response to the fifth phase offset target is actually occurring before the fourth offset target. This is most likely to be caused by the subject attending just the falling edge of the phase segment four stimulus and the rising edge of the phase segment five stimulus resulting in a shared predicted phase angle for these two targets. In contrast, Figure 4(b) the predicted phase angles for the method B occur in the expected order and are distributed with larger equality and ties in with the mean recorded phase separation $\bar{\phi}$ between the methods as depicted in Table 1 .

\section{Discussion and Conclusion}

The findings in this paper may not apply solely to SSVEP BCI's utilizing phase information. In fact the results here are also relevant to traditional magnitude domain paradigms in that SSVEP amplitude will be reduced if phase distribution of the single cycle vector components is inconsistent. This issue has likely remained hidden in the magnitude domain as vectors separated by less than $90^{\circ}$ still remain additive meaning that cancellation effects on overall magnitudes would appear at stimuli larger than $15 \mathrm{~Hz}$, which are less employed in current SSVEP BCI.

While it must be said that if a subject was able to be consistent in the inconsistency in regards to which edge of the transition that is attended while using the Method A, then classification accuracy would not be impacted. More likely though that the inconsistency is in reality an artifact of the single frame duty cycle method and cannot be willfully controlled by the subject or fall into a regular subconscious pattern.

It would seem that choice of stimulation duty cycle method so far in SSVEP BCI's has been arbitrary (approximately half using single frame method, half using 50\% duty cycle method) perhaps because of utilizing existing code from other paradigms or other reasons. The results presented in this 
paper offer compelling motivation to employ a 50\% duty cycle especially in the case of phase discriminating SSVEP BCIs.

\section{Acknowledgement}

The first author was funded by Engineering and Physical Sciences Research Council studentship.

\section{References}

[1] R. Acharya, E.C.P. Chua, K.C. Chua, L.C. Min, and T. Tamura, "Analysis and automatic identification of sleep stages using higher order spectra," International Journal of Neural Systems, vol. 20, no. 6, pp. 509-521, 2010.

[2] T. Balli and R. Palaniappan, "On the complexity and energy analyses in EEG between alcoholic and control subjects during delayed matching to sample paradigm," International Journal on Computational Intelligence and Applications (special issue on Biomedical Signal Sensing and Intelligent Information Processing), vol. 7, no. 3, pp. 301-315, 2008.

[3] R. Palaniappan, "Identifying individuality using mental task based brain computer interface," Proceedings of 3rd International Conference on Intelligent Sensing and Information Processing, Bangalore, India, pp. 239-242, 13-17 December, 2005.

[4] P. Goel, H. Liu, D. Brown, A. Datta, "On the use of spiking neural network for EEG classification." International Journal of Knowledge-Based and Intelligent Engineering Systems, vol. 12 (4), pp. 295-304, 2008.

[5] K.V.R. Ravi, R. Palaniappan, S.-H. Heng, "Simplified fuzzy ARTMAP classification of individuals using optimal VEP channels," International Journal of Knowledge-Based and Intelligent Engineering Systems, vol. 10 (6), pp. 445-452, 2006.

[6] W. Hsu, "Continuous EEG signal analysis for asynchronous BCI application," International Journal of Neural Systems, vol. 21 (4), pp. 335-350, 2011.

[7] F. B. Vialatte, M. Maurice, J. Dauwels and A. Cichocki, "Steady-state visually evoked potentials: focus on essential paradigms and future perspectives," Progress in Neurobiology, vol. 90 (4), pp. 418-438, 2010.

[8] C. N. Gupta, Y. U. Khan, R. Palaniappan, and F. Sepulveda, "Wavelet framework for improved target detection in oddball paradigms using P300 and gamma band analysis," special issue on Biosignals: Data Acquisition, Processing and Control, International Journal of Biomedical Softcomputing and Human Sciences, vol.14, no. 2, pp.61-67, 2009.

[9] M. Bach, T. Meigen, and H. Strasburger, "Raster-scan cathode-ray tubes for vision research limits of resolution in space, time and intensity, and some solutions," Spatial Vision, vol. 10 (4), pp. 403 414, 1997.

[10] M. Bach and T. Meigen, "Do's and don'ts in Fourier analysis of steady state potentials," Documenta Ophthalmologica, vol. 99 (1), pp. 69-82, 1999.

[11] J. J. Wilson and R. Palaniappan, "Analogue mouse pointer control via an online steady state visual evoked potential (SSVEP) brain-computer interface," Journal of Neural Engineering, vol. 8(2), doi: $10.1088 / 1741-2560 / 8 / 2 / 025026$.

[12] T. Kluge and M. Hartmann, "Phase coherent detection of steady-state evoked potentials: Experimental results and application to brain computer interfaces," Third International IEEE/EMBS Conference on Neural Engineering, Hawaii, USA, pp. 425-429, 2007.

[13] C. Jia, X. Gao, B. Hong and S. Gao, "Frequency and phase mixed coding in SSVEP-based brain-computer interface," IEEE Transactions on Biomedical Engineering, vol. 58 (1), pp. 200-206, 2011.

[14] P. L. Lee, J.J. Sie, Y.J. Liu, L.H. Wu, M.H. Lee, C.H. Shu, P.H. Li, C.W. Sun and K.K. Shyu, "An SSVEP-actuated brain computer interface using phase-tagged flickering sequences: a cursor system," Annals of Biomedical Engineering, vol. 38 (7), pp. 2383-2397, 2010.

[15] D. Zhu, J. Bieger, G. C. Molina and R. M. Aarts, "A survey of stimulation methods used in SSVEP-based BCIs," Computational Intelligence and Neuroscience, article ID 702357, 12 pages, 2010.

[16] J. J. Wilson and R. Palaniappan, "Augmenting a SSVEP BCI through single cycle analysis and phase weighting," 4th International IEEE EMBS Conference on Neural Engineering, Antalya, Turkey, pp. 371-374, 2009.

[17] R. A. Dobie and M. J. Wilson, "Analysis of auditory evoked-potentials by magnitude-squared coherence," Ear and Hearing, vol. 10 (1), pp. 2-13, 1989. 
[18] J. R. Wolpaw, N. Birbaumer, W. J. Heetderks, D. J. McFarland, P. H. Peckham, G. Schalk, E. Donchin, L. A. Quatrano, C. J. Robinson, and T. M. Vaughan "Brain-computer interface technology: A review of the first international meeting," IEEE Transactions on Rehabilitation Engineering, vol. 8, no. 2, pp. 164-173, 2000. 
Table 1. Comparison of methods by MSC, mean $\bar{\phi}$ and ITR per subject.

\begin{tabular}{|c|c|c|c|c|c|c|}
\hline & \multicolumn{3}{|c|}{ Method A } & \multicolumn{3}{|c|}{ Method B } \\
\hline & $\begin{array}{l}\text { Mean } \\
\text { MSC }\end{array}$ & Mean $\bar{\phi}\left(^{\circ}\right)$ & ITR (bits/min) & $\begin{array}{l}\text { Mean } \\
\text { MSC }\end{array}$ & Mean $\bar{\phi}\left({ }^{\circ}\right)$ & ITR (bits/min) \\
\hline S1 & 0.15 & 65.65 & 34.45 & 0.16 & 50.43 & 46.94 \\
\hline S2 & 0.17 & 48.93 & 26.54 & 0.15 & 42.28 & 63.82 \\
\hline S3 & 0.22 & 36.48 & 63.82 & 0.19 & 34.81 & 71.52 \\
\hline $\mathrm{S} 4$ & 0.18 & 47.45 & 39.28 & 0.16 & 32.45 & 91.99 \\
\hline S5 & 0.15 & 49.21 & 29.85 & 0.15 & 44.56 & 63.82 \\
\hline & 0.17 & 49.54 & 38.79 & 0.16 & 40.91 & 67.62 \\
\hline
\end{tabular}



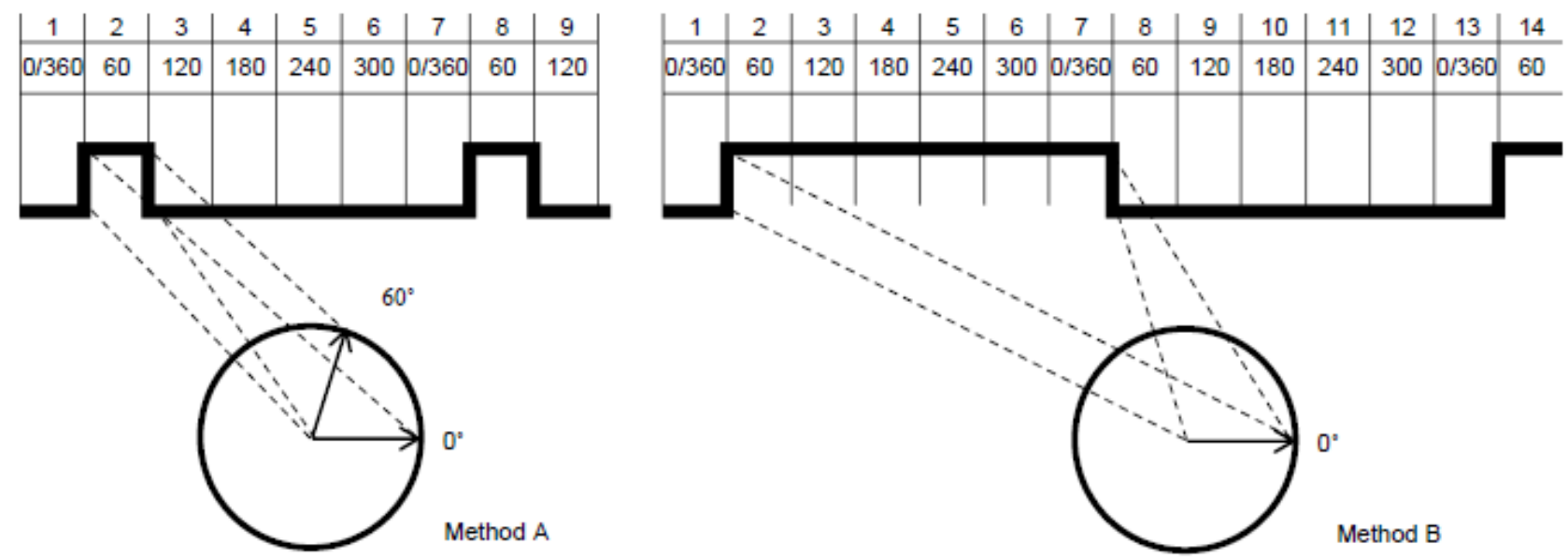

Figure 1. Alternative methods for stimulus construction for an example of $12 \mathrm{~Hz}$ alternation rate. Method A -Single frame duty cycle (left). Method B-50\% duty cycle (right). The top lines display the frame number and corresponding phase offset. 

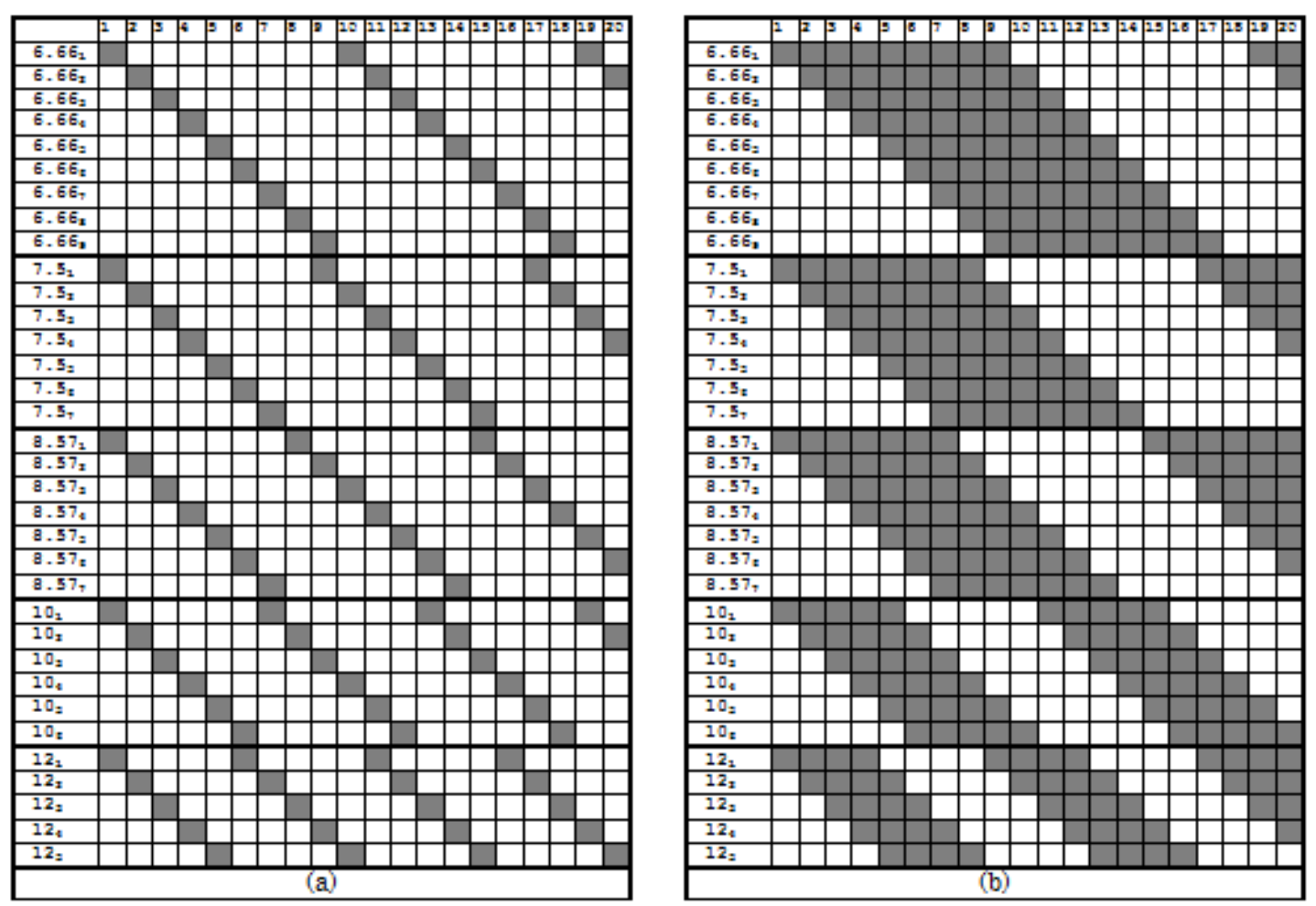

Figure 2. Alternative stimulation paradigms. (a): single frame alternation and (b): $50 \%$ duty cycle. Columns represent VDU frames and rows represent targets (frequency ${ }_{\text {phase offset). The shade represents }}$ the luminosity of the stimulus at that particular frame and target. 


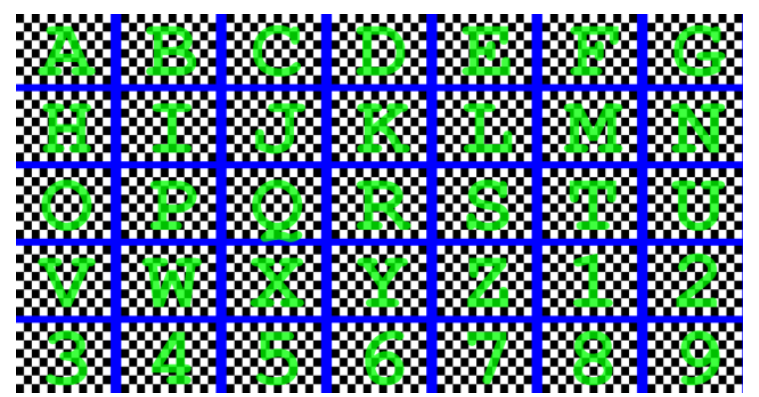

Figure 3. Stimulation screen displaying 35 alternating checkerboard targets. 


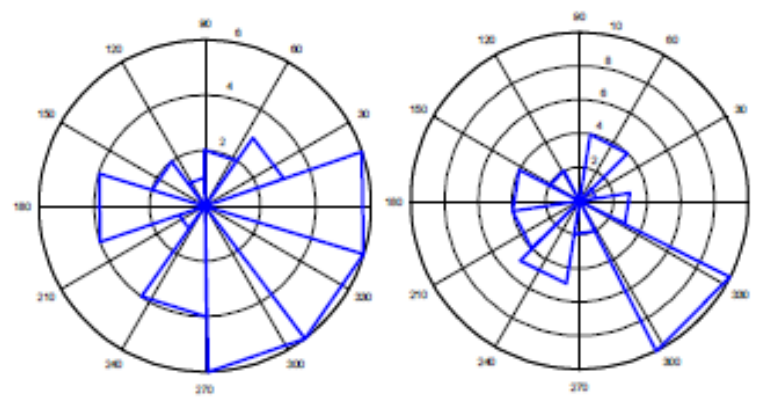

(a)

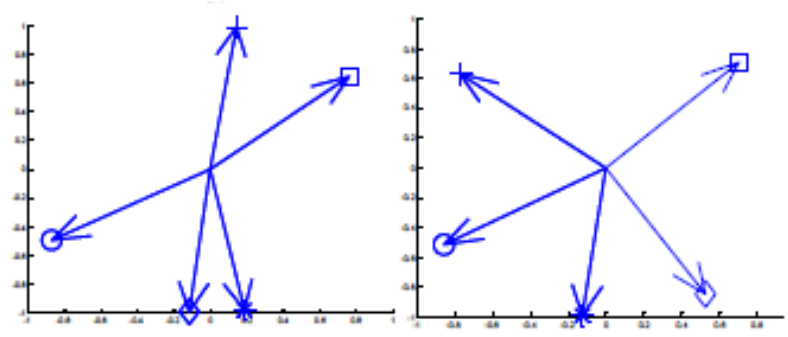

(b)

Figure 4(a): Phase histograms Method A (left) and Method B (right) for $12 \mathrm{~Hz}$ stimulus, phase five (corresponding to diamond vector in Figure 4(b)), (b): Phase calibration maps Method A (left) and Method B (right) for $12 \mathrm{~Hz}$ stimuli. Phase stimulation true order $=0+\square \diamond *$. 


\section{Reply to reviewer comments}

The authors thank the reviewers for the comments. Please find below our responses detailing the changes made.

\section{Comment}

An explicit aim of the study and the paper layout should be provided in Introduction

\section{Reply}

Description on the aim has been included in the abstract and introduction. In addition, more details have been included in the introduction on BCI. Paper layout has also been included in the Introduction.

\section{Comment}

Repetitions like the list of frequencies on page 3 should be avoided.

\section{Reply}

Repetition removed.

\section{Comment}

Originally announced integer divisors of $60 \mathrm{~Hz}$ are not followed; why?

\section{Reply}

The used frequencies $(6.66 \mathrm{~Hz}, 7.5 \mathrm{~Hz}, 8.57 \mathrm{~Hz}, 10 \mathrm{~Hz} \& 12 \mathrm{~Hz})$ are still integer divisions of $60 \mathrm{~Hz}$. The frequencies mentioned in section 2, page 2 are just examples. More details on these have been included in Section 2.

\section{Comment}

What is available phase offset mentioned under Stimulating Engine

\section{Reply}

This has been removed and discussion on 'phase' can be found in Section 4.2.

\section{Comment}

- N above Eqn (1) should have argument $\mathrm{f}$ (similarly as \Delta\phis;

moreover, later on $\mathrm{N}$ is used for the number of targets: are these numbers really identical?

\section{Reply}

Argument $f$ has been included for $\mathrm{N}$ and delta \phis. To avoid confusion, this target frequency function has been changed to $M$, keeping $N$ to represent number of targets. 


\section{Comment}

Subjects undergoing the tests should be characterized a bit more as it can be expected that age, education, sex etc. could influence the results

\section{Reply}

Since all subjects completed both Method $A$ and Method $B$ and comparison of performance was for each subject, the mean age, sex, formal education, etc do not affect the comparative results. Details about this have been included in the revised paper.

\section{Comment}

The experimental conditions are described but their choice seems to be a bit arbitrary; at least some discussion of this choice is desirable

\section{Reply}

Frequency tagged flashing SSVEP stimuli are typically generated by consistently varying the luminance of a target for a set number of frames or display events. In published literature there are two main modalities of flash generation as outlined in 2.1 and 2.2 and the experiment conditions were designed to stimulate these two modalities.

\section{Comment}

Unnecessary abbreviations should be avoided (like single occurrence of VEP at the beginning of Section 4)

\section{Reply}

Done.

\section{Comment}

Subscript $N$ in (3) should probably be $n=1, \ldots, N$; moreover $N$ is introduced to late (under Eqn (4)) and it is in the clash with $N(f)$ in Eqn (1)

\section{Reply}

Subscript N has been corrected. To avoid confusion, target frequency function (in Equation 1 ) has been changed to $M$, keeping $N$ as the number of targets.

\section{Comment}

Neighboring phase offset (beginning of Sec 4.2) is undefined and unknown to nonexperts

\section{Reply}

We have revised the Section, which hopefully is clearer. 


\section{Comment}

$P$ under Information Transfer Rate is undefined

\section{Reply}

$P$ is the classification accuracy rate and has been defined above Eq. (5).

\section{Comment}

Eqn (5) looks strange: it resembles "spoilt" entropy; please, motivate or refer to the used source

\section{Reply}

It is a standard equation used in BCI studies. A reference has been cited.

\section{Comment}

- In Section 5 make cross-reference to definitions of the used characteristics (MSC, $\backslash$ bar $\{\backslash p h i\}$, ITR. Moreover, explain what $(p=0.009, p=0.018)$ mean: not everybody has it as standard

\section{Reply}

Cross reference to section 4 has been included. The t-test significance ( $p$ value) has been described as such.

\section{Comment}

- Are the discussed results (Figure 4) typical? Figure itself has a poor graphics and its information content is well hidden

\section{Reply}

The figure is typical for such studies.

\section{Comment}

- Reference to Figure 3 occurring after discussion of Figure 4 seems to refer to something else - the presentation is mixed up

\section{Reply}

Reference to the figures has been corrected as appropriately.

\section{Comment}

- Si in Table 1 is an example of non-introduced symbol

\section{Reply}

Si is described in eq (4). 